\title{
Stretch-Induced Alternation of Cardiac Action Potential Duration: A Study of Computer Simulations
}

\author{
JC Hsieh \\ Department of Bioinformatics, Chung Hua University, Hsin-Chu, Taiwan
}

\begin{abstract}
Stretch-activated channels (SACs) have been considered as one of the possible modulators on the mechanisms of mechano-electrical feedback (MEF) in the heart. With the abilities to modulate action potential duration (APD) during the systolic period and to trigger action potentials directly by diastolic stretch, SACs could play an important role on stretch-induced arrhythmias. The objective of this present study was to explore the impact of SACs on modulating action potential duration by different kinds of stretches including slow ramp stretch and quick step stretch. A mathematical model of ventricular cells based on Noble's descriptions was constructed with the addition of the time-independent model of SACs. The differential equations involved in the cell model were solved numerically by the algorithm of Runge-Kutta-Fehlberg with adaptive time steps. The simulation results indicated the following. First, the action potential duration was shortened 3\% to $8 \%$ without crossover with non-stretched action potential under various slow ramp stretches. Second, by quick step stretch, the lengthening of action potential duration was observed with crossover with non-stretched action potential at certain membrane potentials. The crossover potentials varied from $-18 \mathrm{mV}$ to $-50 \mathrm{mV}$ with increased sustained quick step stretch from sarcomere length 1.1 to 2.3. Third, the presence of a specific time window allowed shortening action potential duration if quick step stretch applied inside. The window duration was increased with the amplitude of stretch. It was concluded that SACs activated by ramp stretch could lead to the shortening of action potential duration independent of the activation timing. However, the shortening or lengthening of action potential duration was determined by activation timing under quick step stretch.
\end{abstract}

\section{Introduction}

The SACs have been recognized as one of the possible pathways to mechanoelectrical feedback on the heart [1]. The MEF can provide reasonable explanations on newly observed stretch-induced arrhythmias [2], which might open an avenue for the design of new anti-arrhythmic agents. With computer simulations of SACs, the dynamics of SACs can be studied, which also provides insights into experimental electrophysiology of SACs. My previous simulation studies showed that blocking IK1 channels could either promote or prevent stretch-induced arrhythmias [3,4]. Sustained stretching the cardiac ring muscle could increase the ratio of excitation gap to action potential duration, which leads to the generation of double-wave reentry [5]. Other recent studies indicated that the activation timing of SACs can induce controversy responses of action potential duration [6] and that interactions of SACs between specific ion channels such as calcium can modulate the response of action potential [7]. Despite of recent progress on electrophysiology of SACs, the force transduction through SACs and the modulation of SACs on action potential remain unclear. In this study, the effects of SACs on modulating action potential duration by two different kinds of stretches including slow ramp stretch and quick step stretch were investigated, and the results of simulations could provide insight into the complex dynamics of SACs. A mathematical model of ventricular cells based on Noble's descriptions was constructed with the addition of the time-independent model of SACs [8]. The differential equations involved in the cell model were solved numerically by the algorithm of Runge-Kutta-Fehlberg with adaptive time steps.

\section{Methods}

A mathematical model of ventricular cells based on Noble's descriptions [8] was adapted with the addition of the time-independent model of SACs. The differential equations involved in the cell model were solved numerically by the algorithm of Runge-Kutta-Fehlberg with adaptive time steps.

\subsection{SACs model}

The mathematical modelling of SACs was based on the experimental observations directly with their time- 
independent dynamics, therefore equation (1) [6] was usually adopted as simulation of SACs.

$$
\begin{aligned}
& I_{s a c}=g_{s a c} f_{s}\left(V-V_{s a c}\right) \\
& f_{s}=\frac{1}{1+k e^{-r\left(l-l_{0}\right)}}
\end{aligned}
$$

Where $I_{s a c}$ represents the current of SACs, $g_{s a c}$ represents the maximum conductance of SACs, $f_{s}$ represents stretch-dependant regulator of maximum conductance and its values is between 0 and $1, V$ represents membrane potential, $V_{s a c}$ represents the reversal potential of SACs, $k$ represents the user-defined equilibrium constant, $r$ represents the user-defined sensitive parameter, $l$ represents the current sarcomere length and $l_{0}$ represents the resting sarcomere length.

\subsection{Pacing protocol}

The S1-S2 electrical stimuli were paced to invoke action potential, followed by a premature stretch stimulus during the second stimulus. Two kinds of stretches including quick step stretch and slow ramp stretch were used to investigate their effects on action potential duration.

\section{Results}

\subsection{The effects of quick step stretch on APD}

With the application of quick step stretch on the SACs, the alternations of APD were measured. In the following simulations, the interactions between activation time and the stretched length of SACs could play an important role on modulating APD.

In Figure 1, the APD was prolonged when sacromere length was stretched to 1.6 from resting length of 1.5 and the activation time of SACs was set as the same time as the second electrical stimulus. The crossover of action potential with non-stretched one was observed at membrane potential at $-19 \mathrm{mV}$.

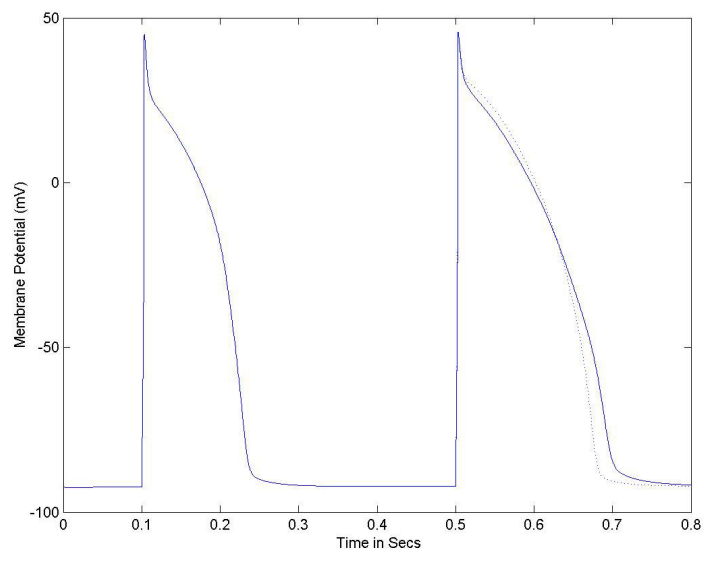

Figure 1: APD was lengthened by a sustained quick step stretch slightly, and SACs were activated on the initial time of the second stimulus.

The crossover of APD with non-stretched one at membrane potential $-70 \mathrm{mv}$ was shown in Figure 2. In this simulation, the sarcomere length was lengthened to 2.3 and SACs were activated at the initial time of the second electrical stimulus. The APD was shortened at membrane potential $-60 \mathrm{mv}$, but it was prolonged slightly at membrane $-70 \mathrm{mv}$.

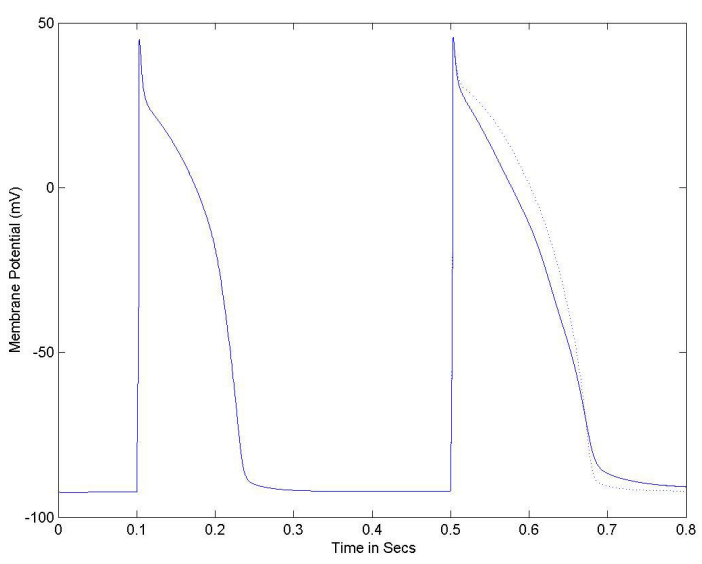

Figure 2: The crossover of APD with non-stretched one was seen by a severe quick stretch, but the APD was shortened at APD-60.

By manipulating the activation time of SACs, the APD was measured. In Figure 3, the simulation indicated that the APD was shortened if the activation time of SACs was delayed to the middle of systolic period. In this simulation, the sacromere length was stretched to 1.6. 


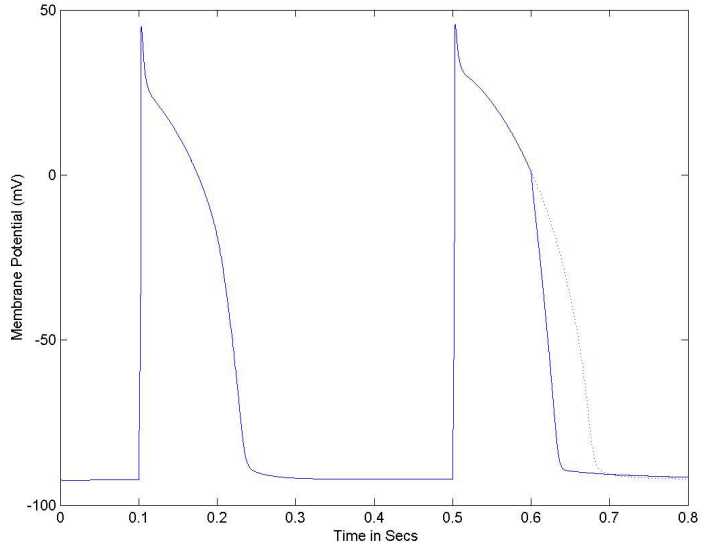

Figure 3: APD was shortened if the activation time of SACs was delayed during systolic period.

A series of two stretches were applied onto the SACs, and the APD was shortened as shown in Figure 4. In this simulation, the first severe stretch was applied onto the beginning of S2 at sacromere length 2.2, while the second slight stretch with sarcomere length 1.6 was applied onto the middle of systolic period.

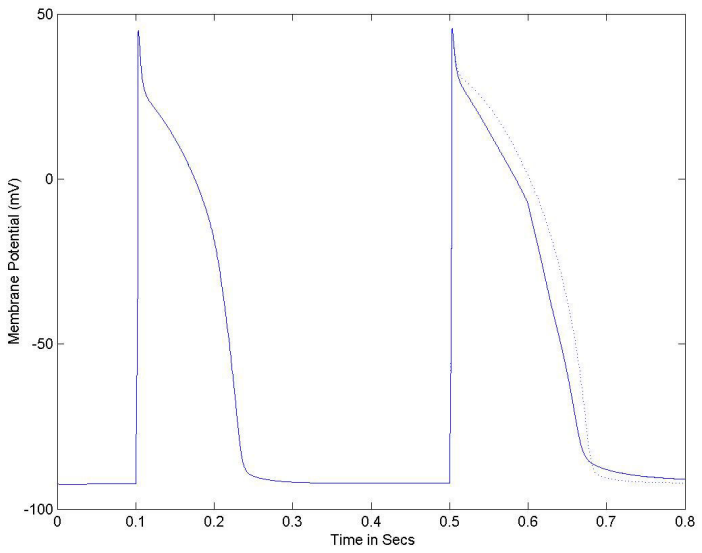

Figure 4. APD was shortened by a two series of quick systolic stretches.

\subsection{The effects of slow ramp stretch on APD}

In the following simulations, a slow ramp stretch was applied during the S2 stimulus to test its effects on APD.

A slightly shortened APD was shown in Figure 5, when stretch was lengthened from 1.2 to 2.2 during the initial $100 \mathrm{msec}$ of S2. As Figure 6 shows, by reducing the ascending rate of SACs, the shortened APD was observed clearly if the same stretch was applied for 300 msec.

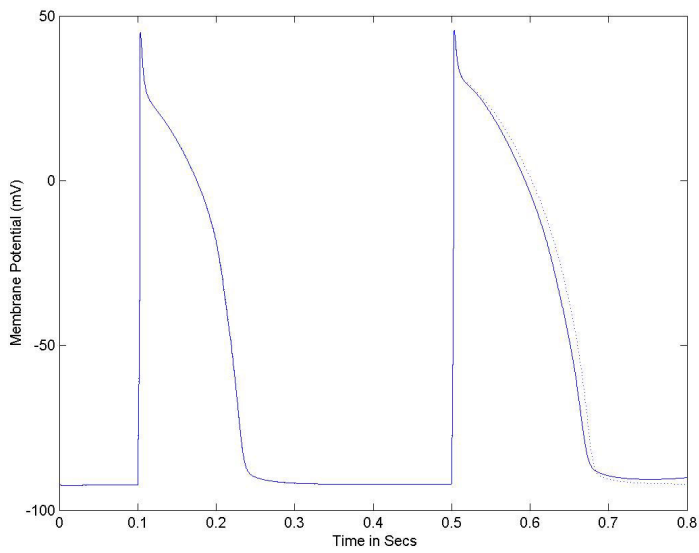

Figure 5: APD was shortened slightly by a slow ramp stretch in a severe stretch status.

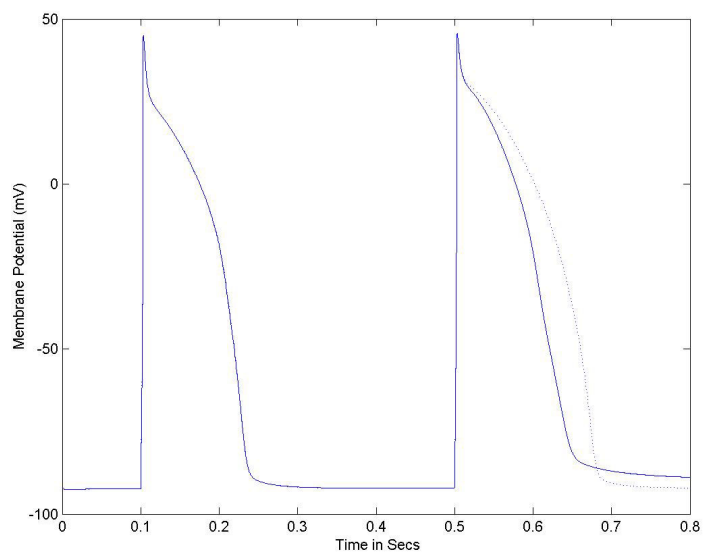

Figure 6: A slower ramp stretch in a severe stretch status was used to induce shortened APD.

In Figure 7, a mild stretch was applied to test the changes of APD. The results indicated that APD was shortened to some extent compared with non-stretched APD. In this simulation, the stretch was lengthened from 1.2 to 1.6 during the initial $100 \mathrm{msec}$ of S2. In Figure 8, APD was changed clearly if the ramp stretch was prolonged to $300 \mathrm{msec}$ with the same stretch as shown in Figure 7. 


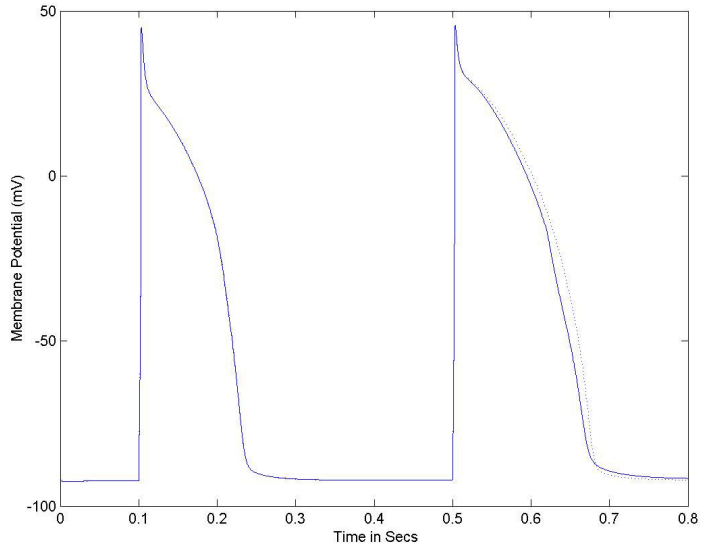

Figure 7: APD was not changed much by a mild stretch.

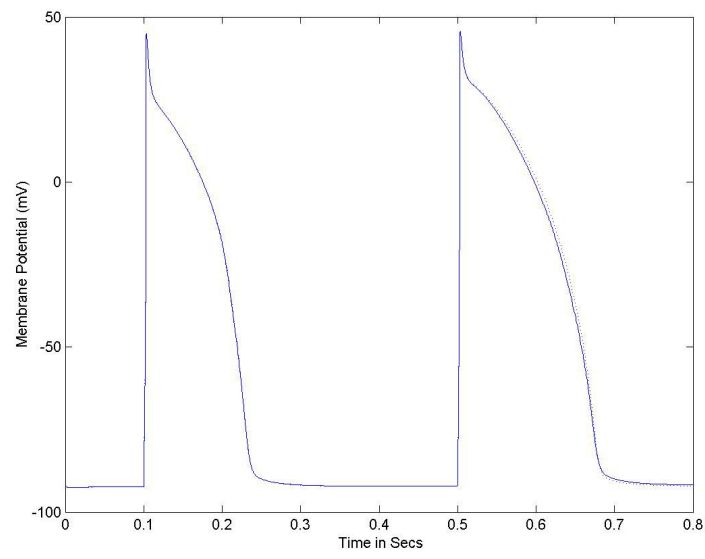

Figure 8: APD was changed by a slower ramp stretch in a mild status.

\section{Discussions}

The simulation results showed that quick step stretch and slow ramp stretch could have different physiological effects on ventricular cells. In the simulations shown in Figures 6 to 8 , the action potential duration was shortened $3 \%$ to $8 \%$ without crossover with non-stretched action potential under various slow ramp stretches. The shortening degree appeared independent of activation time of SACs but it seemed related to the interactions between the ramp slope and the stretched length. In the simulations of quick step stretch shown in Figures 1 to 4, the lengthening of action potential duration was observed with crossover with non-stretched action potential at certain membrane potentials. The crossover potentials varied from $-18 \mathrm{mV}$ to $-50 \mathrm{mV}$ with increased sustained quick step stretch from sarcomere length 1.1 to 2.2 . The presence of a specific time window allowed shortening action potential duration if quick step stretch applied inside. The window duration was increased with the amplitude of stretch.

\section{References}

[1] Craelius W. Stretch-activation of rat cardiac myocytes. Exp Physiol. 1993; 78(3):411-23.

[2] Akay M, Craelius W. Mechanoelectrical feedback in cardiac myocytes from stretch-activated ion channels. IEEE Trans Biomed Eng. 1993; 40(8):811-6.

[3] Hsieh JC , Craelius W. Excitation of ventricular mo0del myocytes by stretch-activated currents. Computers in Cardiology 1998; 25: 569-572.

[4] Hsieh JC, Chen SA. The Role of Stretch-Activated Channels on Antiarrhythmic Therapies: Computer Simulations. Computers in Cardiology 2001; 28:545-548.

[5] Hsieh JC, Craelius W. Termination and acceleration of ventricular tachycardia in a propagating model. Computers in Cardiology 1998; 25: 13-16.

[6] Kohl P, Day K, Noble D. Cellular mechanism of mechanoelectrical feedback in a mathematical model Can J. Cardiol. 1998; 111-119.

[7] Kohl P, Sachs F. Mechano-electrical Feedback in cardiac cell. Phil. Trans. R. Soc. Lond. 2001;359:1173-1185.

[8] Noble D, Varghese A, Kohl P, Noble P. Improved guineapig ventricular model incorporating a dyadic space, Ikr, Iks and length and tension dependant processes. Can J. Cardiol. 1998; 14(1) 123-134.

\section{Acknowledgements}

This study was fully sponsored by Chung Hua University, Hsin-Chu, Taiwan.

Address for correspondence.

Jui-chien Hsieh, Ph.D.

Department of Bioinformatics

Chung Hua University

30 Tung Shiang, Hsin-chu 30067

Taiwan

E-mail address: jchsieh@chu.edu.tw 